\title{
BASIC BIOLOGICAL SCIENCES
}

\section{Synchronized Crystal Dissolution Behavior for Tooth Enamel and Synthetic (NBS) Hydroxyapatite}

\author{
M. B. Fawzi, T. Sonobe, W. I. Higuchi, and J. J. Hefferren* \\ College of Pharmacy, University of Michigan, Ann Arbor, Michigan 48109, USA
}

The synchronized crystal dissolution hypothesis previousiy proposed to explain the unusual dissolution behavior of human dental enamel and hydroxyapatite in partially saturated acidic media has been critically examined with disso. lution-dialysis transport experiments. The findings are in accord with the hypothesis. A model based upon a variable effective solubility for the hydroxyapatite crystal is proposed.

Recent studies of human dental block enamel and synthetic hydroxyapatite (HAP) pellet dissolution under high partial saturation conditions have revealed a very unusual dissolution pattern. ${ }^{1}$ Instead of a linear amount versus time dissolution relation, which is the usual case, $^{2,3}$ a repetitive stepwise dissolution pattern has been observed for which no parallel in the literature has been found.

It is believed that the unusual dissolution process is the key to many of the unanswered questions about HAP, including those related to the peculiar solubility behavior (slurry density effect, for example) . ${ }^{4-6}$ It is also probably basic to the understanding of the histology of incipient caries and to the "white spot" formation phenomenon ${ }^{z}$ since the conditions for both are very similar.

A possible mechanism was proposed ${ }^{8}$ to explain these results. It is based upon a hypothesis that the HAP crystals may dissolve in a synchronized fashion (that is, "in phase") when the ambient solution ion activity product can oscillate about a critical value, the oscillations resulting from bursts of dissolution from the

This investigation was supported by USPHS Grant DE-01830 from the National Institute of Dental Research, National Institutes of Health, Bethesda, Md.

Received for publication August 19, 1975 .

Accepted for publication July 28, 1976.

* Dr. Hefferren is also associated with the Research Institute, American Dental Association Health Foundation. crystals followed by diffusional relaxation of the ambient ions into the bulk solution. The key to this concept resides in the picture that there are substantial "solubility" gradients in domains of the order of unit cell dimensions in the direction of dissolving crystals. Thus, when the ambient solution conditions are appropriate, the rapidly dissolving crystals may be slowed up, whereas the slowly dissolving ones are permitted to catch up and the assembly of crystals becomes synchronized. Then when the ambient solution activity product drops below a critical value, all crystals in the population dissolve the domainal increment simultaneously, again raising the ambient ion activity product to a value greater than the critical value.

It is the purpose of this communication to present the results of the critical investigation of the aforementioned hypothesis by an independent set of studies in which a suspension of HAP crystals in a dialysis bag is placed in a partially saturated acetate buffer similar to those used in the dissolution studies. The dissolution of HAP crystals and the diffusion of ions out of the bag is followed as a function of time by analyzing the ambient solution ion concentration over suitable time intervals. The results of these studies have dramatically supported the synchronous dissolution hypothesis.

Theory and Rationale For THE EXPERIMENTS.-Figure 1 shows schematically the proposed model. The activity products, $\mathrm{pK}_{\mathrm{HAP1}}$ and $\mathrm{pK}_{\mathrm{HAP2}}$ are the maximum and the minimum surface ion activity products for the HAP crystallite during dissolution, respectively. These are within domains having dimensions of the order of a unit cell (that is, $10^{-3} \mathrm{~cm}$ ).

Synchronization may occur when the solution's ionic activity products adjacent to the crystal surfaces are slightly greater than 


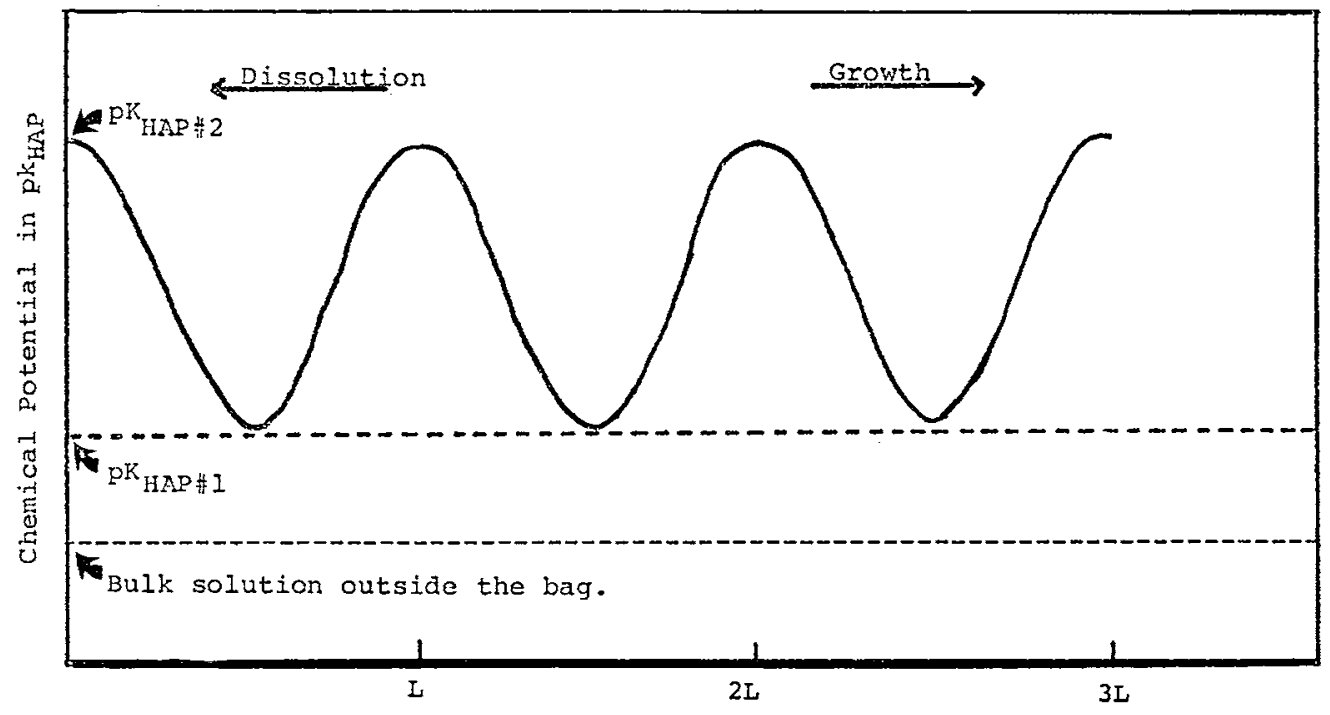

Fig 1.-Possible model for synchronization of HAP crystals (see text). L is of order of magnitude of unit cell dimensions.

$\mathrm{pK}_{\mathrm{HAP1}}$ (the minimum). This ambient solution concentration of ions would then retard or inhibit dissolution from crystals in stages of dissolution where the crystal surface potentials would be greater than but near the ambient solution concentration. The crystals that are "lagging" would generally have higher chemical potentials and these would dissolve and catch up to those that have slowed down, and thus all of the crystals could become in phase or synchronized. The experimental setup is designed in such a way as to allow synchronization to take place and be followed experimentally.

This experiment essentially allows the determination of the ambient or "local" ion concentration in the assembly of crystals. Thus, the analyses of the ion concentrations in the bag as a function of time would be comparable to having a microelectrode in the aqueous pores of the enamel monitoring the unusual dissolution behavior of HAP crystals under these conditions.

\section{Materials and Methods}

Three different samples of synthetic HAP were used in this study. These samples were as follows. Sample A or the so-called authentic (NBS) HAP was prepared and characterized according to the method described by Moreno, Gregory, and Brown ${ }^{9}$ and provided by Dr. P. R. Patel of the National Bureau of Standards, Washington, D.G. This sample was used in all of the experiments conducted in the present study except for a few runs carried out with the other two samples. Sample $B$, the socalled cold (NBS) HAP, was synthesized in our laboratories (T.S.) following the procedure described by Moreno, Gregory, and Brown. ${ }^{9}$ Sample C was the radiolabeled (NBS) HAP. This ${ }^{45} \mathrm{Ca}$-labeled HAP sample was prepared in our laboratories using the procedure developed by Moreno, Gregory, and Brown ${ }^{9}$ using ${ }^{45} \mathrm{Ca}$-labeled calcium oxide as the starting material.

All samples were checked by infrared and elemental analysis. The particle size of these sample was controlled by using USP standard sieves (between 200 and 325 mesh).

Buffer solution preparations and the analytical procedure used in determining the ion concentration have been described previously. ${ }^{10}$

Apparatus.-Figure 2 shows a schematic diagram of the apparatus used for the dialysis studies. The setup consisted of a water-jacketed beaker and a dialysis bag holder-stirring assembly. The temperature of the system was contrclled by circulating water at 30 C. A 600 -rpm synchronous motor and the dialysis bag holder were mounted on a polyethylene cover plate. The dialysis bag holder consisted of seven one-sixteenth-inch gauge stainless steel wires attached to a circular stainless steel ring at the bottom forming a cylindrical framework for the dialysis bag. The stirrer was made of Teflon. Three holes were drilled in the polyethylene 


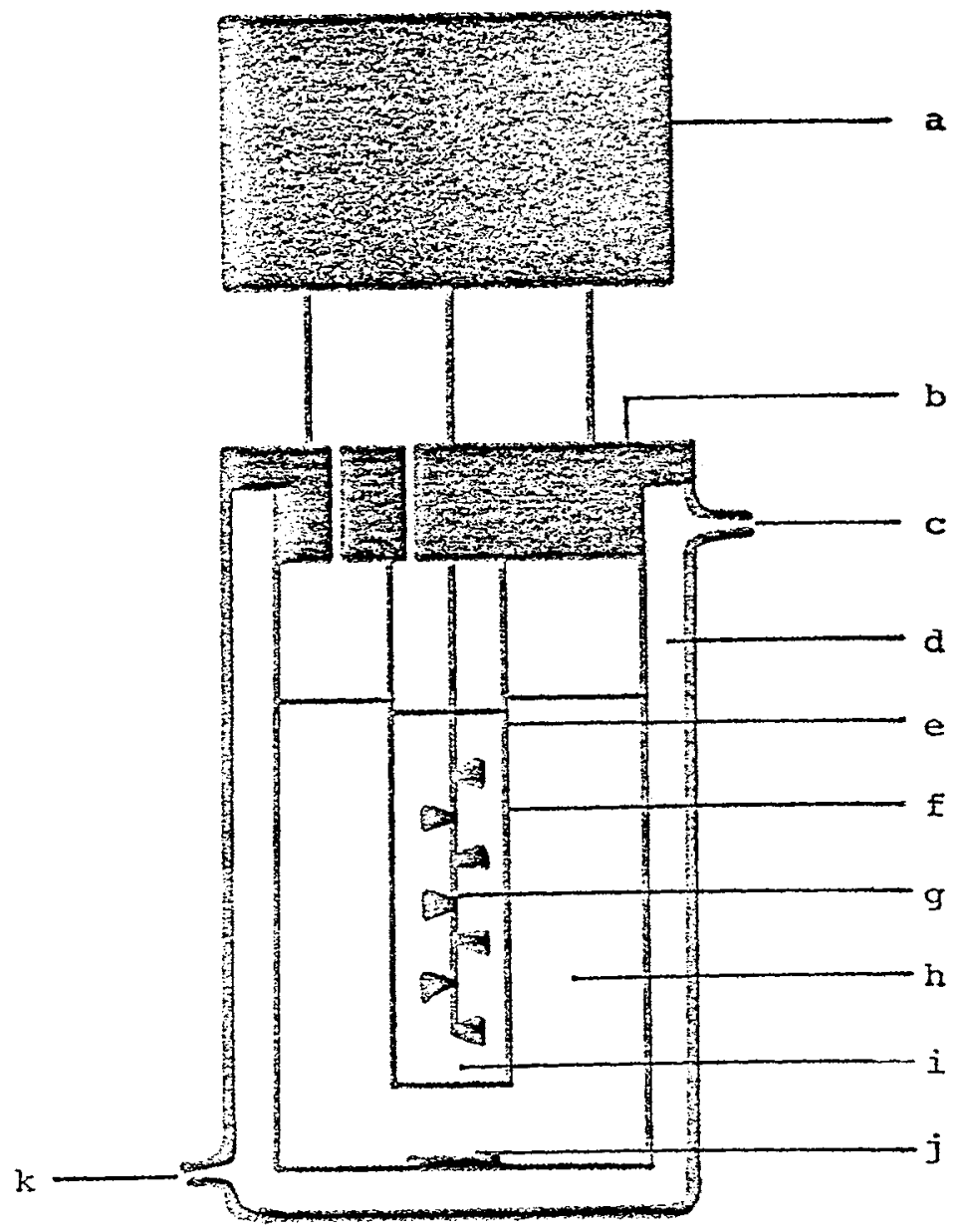

Frg 2.-Dialysis bag assembly. $a$, synchronous motor; $b$, polyethylene cover; $c$, water outlet, $d$, jacketed beaker; $e$, dialysis bag holding framework ; $f$, cellophane dialysis bag; $g$, Teflon stirrer; $h$, partially saturated acetate buffer $\left(\mathrm{pK}_{\mathrm{HAP}}=122.5\right) ; i$, HAP suspension in partially saturated acetate buffer $\left(\mathrm{pK}_{\mathrm{HAP}}=122.5\right) ; j$, stirring bar; $k$, water inlet.

cover plate, one of the stirrer in the center of the cylindrical dialysis bag holder and the other two for sampling inside and outside the bag. The water-jacketed beaker contained $100 \mathrm{ml}$ of the solution which was stirred by a three sixteenths by seven-eighths-inch long Teflon magnetic stirring bar by means of another synchronous motor at $600 \mathrm{rpm}$ at the bottom of the beaker. The dialysis bags* were boiled three times for 30 minutes, each time in double distilled water and then soaked overnight in $0.1 M(\mathrm{pH}, 4.5)$ acetate buffer before using in any experiment. The bags were used only once. The bag's half-lives were within 5 to $10 \%$ of

\footnotetext{
* Union Carbide Corp., Chicago, Il.
}

each other. In the design of this apparatus, the following points were considered important: (1) moderate stirring inside and outside the bag would minimize diffusion layer buildup around the bag and around the HAP particles, and also would keep the HAP particles well dispersed inside the bag; (2) replacement of the samples taken with fresh initial solution would provide constant volume inside and outside the bag and would maintain a constant surface arca-volume ratio of the bag exposed to the outside solution.

EXPERIMENTAL PROCEDURES.--Various slurry densities $(3 \mathrm{mg} / 20 \mathrm{ml}, 6 \mathrm{mg} / 20 \mathrm{ml}, 7 \mathrm{mg} / 20$ $\mathrm{ml}, 8 \mathrm{mg} / 20 \mathrm{ml}, 9 \mathrm{mg} / 20 \mathrm{ml}$, and so on) of 
synthetic HAP ( ${ }^{45} \mathrm{Ca}$-radiolabeled HAP) powder of 200- to 325-mesh particle size were prepared with $20.0 \mathrm{ml}$ of partially saturated acetate buffer $\left(\mathrm{pK}_{\mathrm{HAP}}=122.5\right)$, shaken vigorously for two hours at $30 \mathrm{C}$ or the required temperature, and poured into a dialysis bag whose halflife for calcium ion transport was determined separately. The dialysis bag was immersed in $100 \mathrm{ml}$ of partially saturated acetate buffer $\left(\mathrm{pK}_{\mathrm{HAP}} \simeq 122.5\right)$ at $30 \mathrm{G}$ or the required temperature. Stirring was done both inside and outside of the bag (600 rpm or otherwise mentioned rotation). Samples of $1 \mathrm{ml}$ were with-

$\uparrow$ Millpore Filter Corp., Bedford, Ma. drawn from inside the bag at various intervals by means of a 5-ml hypodermic syringe and needle, and were filtered through Millipore $\dagger$ filters ( 0.22 pore size) contained in Swinnyt filter holders. The solution was replaced by an equal volume of the same buffer solution maintaining a constant volume and by using the same hypodermic syringe and needle. An aliquot of these samples was diluted and analyzed for calcium or phosphate.

In addition to the dialysis studies with the HAP slurries, similar dialysis experiments were carried out with solutions only but under other-

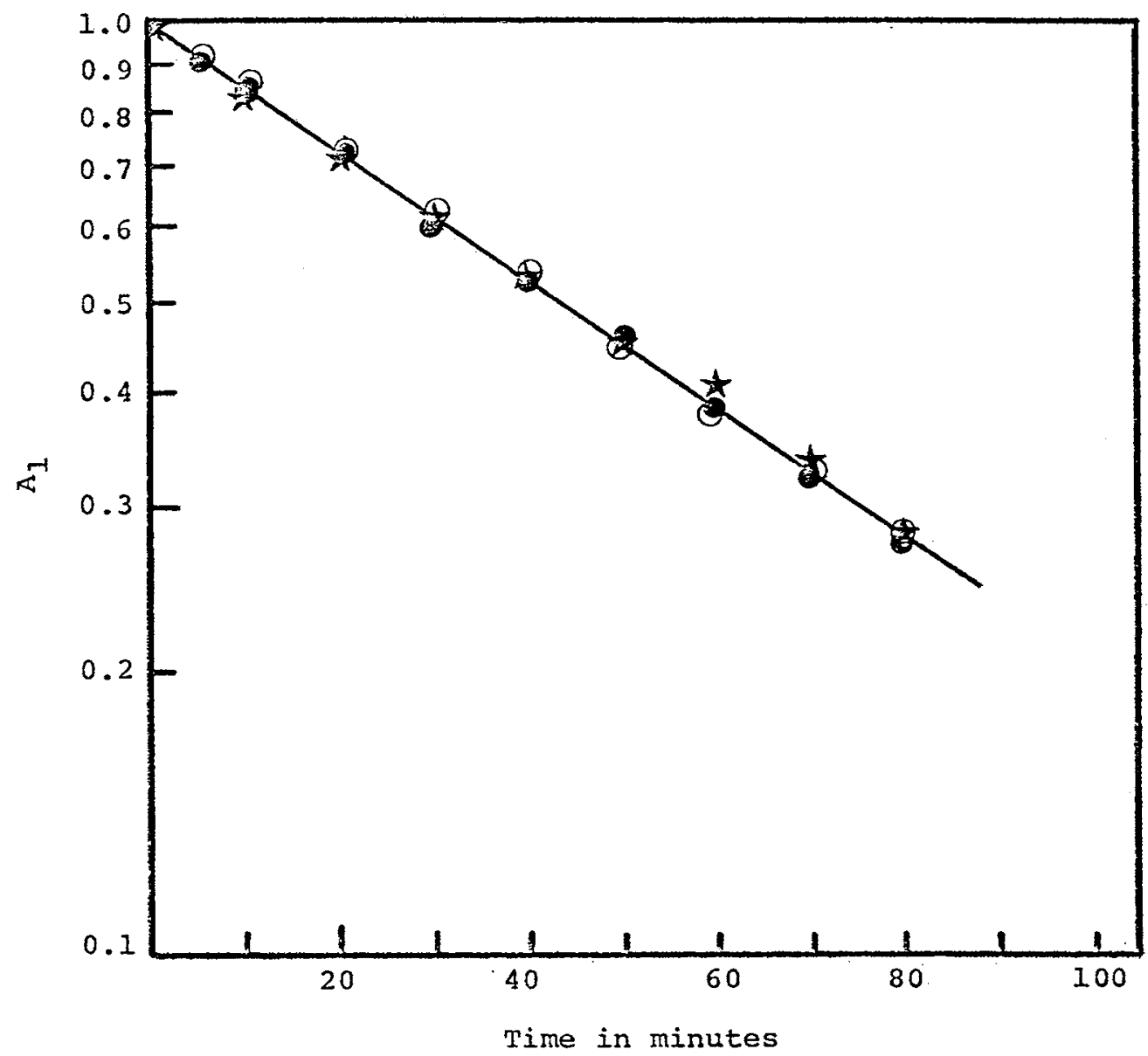

FIG 3.-First order plots used in determination of bag constant for transport of $\mathrm{Ca}^{2}+$ ions through dialysis membrane. All solutions were $0.1 M$ acetate buffer (at $\mathrm{pH}$ of 4.5 ) and $0.5 M$ ionic strength. Solid circle, inside solution was $\mathrm{TCa}=\mathrm{TP}=8.54 \times 10^{-3} \mathrm{M}$ and outside solution was $\mathrm{TCa}=\mathrm{TP}=0$. Solid star, inside

solution was filtrate obtained from suspension prepared with $8 \mathrm{mg}$ HAP added to solution with $\mathrm{TCa}=\mathrm{TP}=8.54 \times 10^{-3} \mathrm{M}$. Outside solution was $\mathrm{TGa}=\mathrm{TP}=0$. Open circle, inside solution was same as in solid star; outside solution was $\mathrm{TCa}=\mathrm{TP}=8.54 \times 10^{-3} M$. 


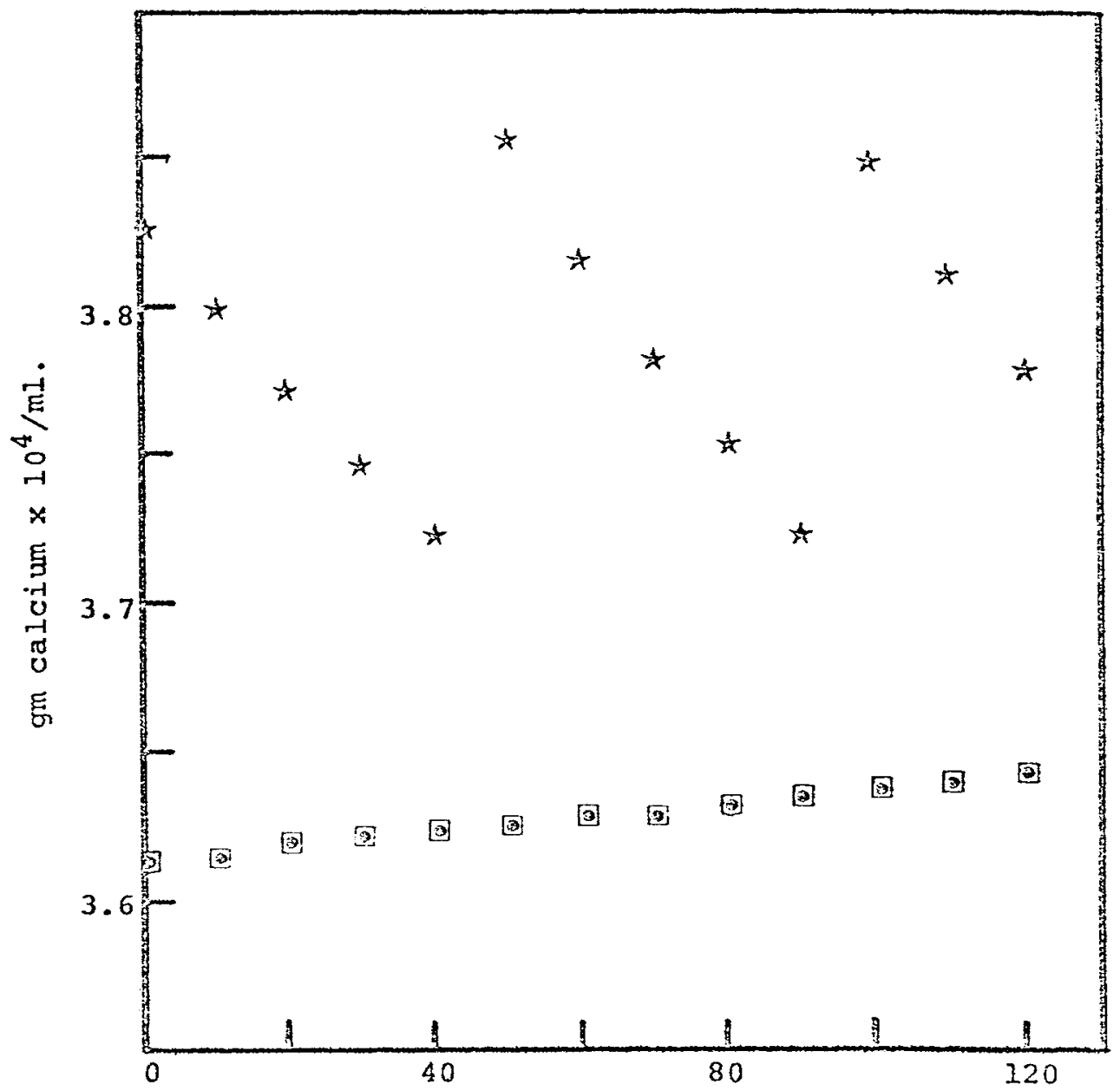

FIG 4.-Dialysis transport-dissolution study of (NBS) HAP (sample A) crystals in partially saturated acidic media. Bulk solution conditions: $0.1 M$ acetate buffer at $\mathrm{pH}$ of 4.5 and

wise identical conditions. These were done for two purposes, to determine the intrinsic cellophane bag transport coefficients and to establish the absence of any unusual transport behavior in the system.

\section{Results and Discussion}

Determination of THE EXPERIMENTAL BAG CONSTANT WITH NO CRYSTALS PRESENT.-In the experimental arrangement, the overall transport of solute into the outside solution involves both the dissolution of HAP crystals and the transfer of the solute across the dialysis bag
$0.5 M$ ionic strength, $\mathrm{TCa}=\mathrm{TP}$. Solid star, inside bag ( $8 \mathrm{mg}$ HAP in $20 \mathrm{ml}$ bulk solution); solid circle in open square, outside bag $(100 \mathrm{ml}$ bulk solution).

membrane. Therefore, it was considered important to first assess the solute transport in the system in the absence of crystals.

The dialysis bag experiment could be considered as a two-compartment system separated by a barrier through which solute can diffuse. The rate of diffusion of solute from one compartment to the other is proportional to the difference in concentration of the two compartments, $\mathrm{C}_{1}$ and $\mathrm{C}_{2}$, and will be from the higher concentration to the lower. In this instance, it will be from the inside to the outside of the bag because of our experimental conditions. There- 
fore, the rate at which the solute leaves the bag is given by equation 1 :

$$
-V_{1} \frac{d C_{1}}{d \mathrm{t}}=S_{b} \cdot P_{b} \cdot C_{1} .
$$

Here, $V_{1}, C_{1}$, and $S_{b}$ are the volume of the solution inside the dialysis bag, the concentration of the solute in the bag, and the surface area of the bag respectively, and $P_{b}$ is the effective permeability coefficient of the bag.

Integration of equation 1 gives

$$
\ln A_{1}=-K \mathrm{t} \text {, }
$$

where $A_{1}$ is the fraction of the amount remaining inside the bag and $K=\frac{S_{b} \cdot \mathrm{P}_{b}}{V_{1}}$. A plot of the
In term in equation 2 versus time should give a straight line. The slope of the straight line would represent $K$ which is the experimental bag constant, and from which we can determine the half-life for the solute decay.

Figure 3 shows the results of bag "control" experiments, that is, runs without HAP crystals in the bag. Two of the runs represent two different solution concentrations inside the bag decaying into a sink solution outside the bag, and one experiment is a saturated solution in the bag decaying into a partially saturated $\left(\mathrm{pK}_{\mathrm{bag}}=122.5\right)$ solution outside the bag. The results for all three experiments are essentially

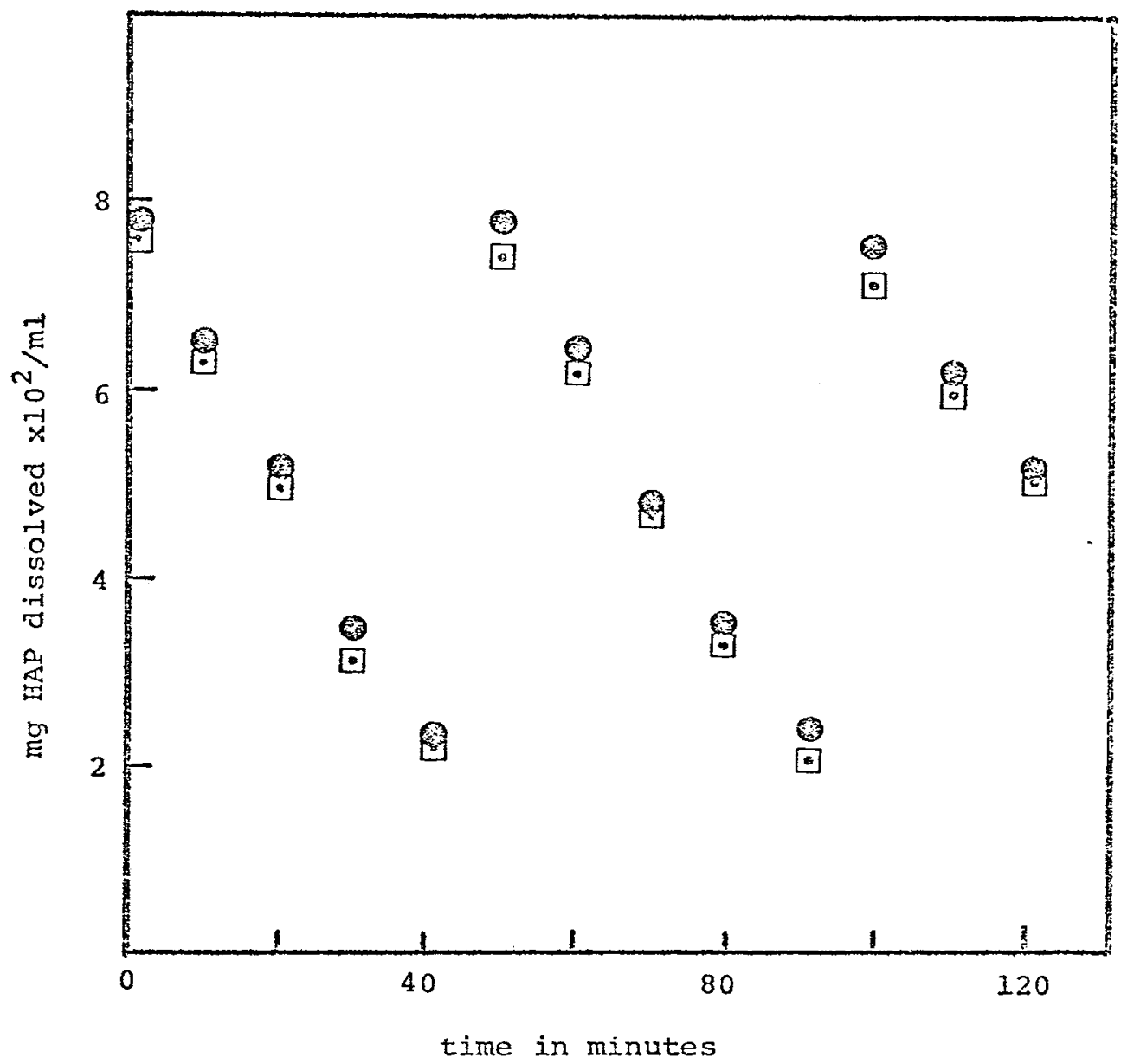

FIG 5.-Dialysis transport-dissolution study of ${ }^{45} \mathrm{Ca}$-HAP (NBS) crystals (sample $\mathrm{C}$ ) in partially saturated media. Bulk solution conditions: $0.1 M$ acetate buffer at $\mathrm{pH} 4.5$ and $0.5 \mathrm{M}$ ionic strength, $\mathrm{TCa}^{45}=\mathrm{TP}=8.536 \times 10^{-3} \mathrm{M}$, with identical specific activity to that of sample $\mathrm{C}$ used. Inside bag ( $8 \mathrm{mg}{ }^{45} \mathrm{Ca}-\mathrm{HAP}$ in $20 \mathrm{ml}$ bulk solution); solid circle, based on ${ }^{45} \mathrm{Ca}$ counts (scintillation method); solid circle in open square, based on phosphate assay (colorimetric method). 


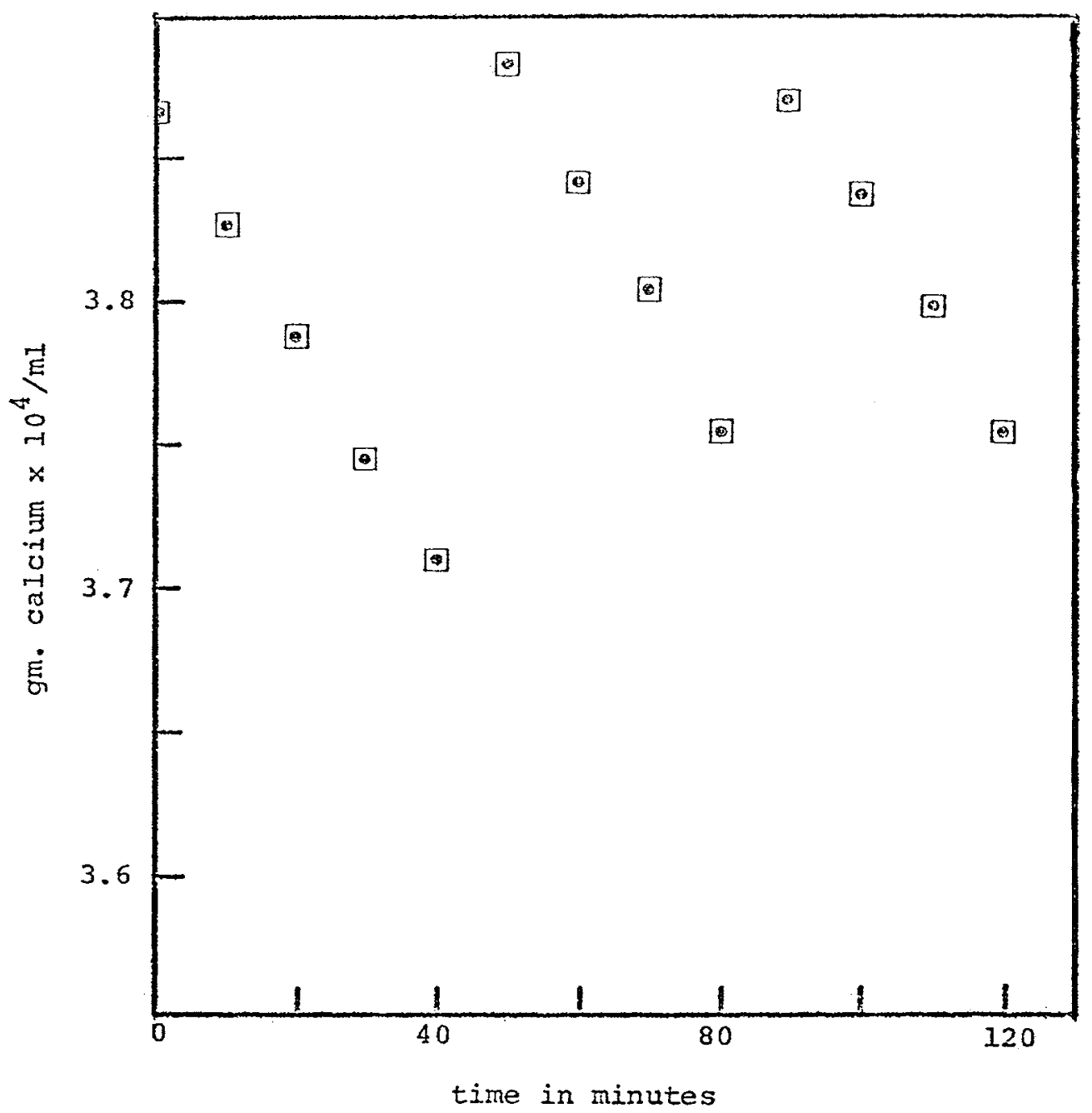

FIG 6.-Dialysis transport-dissolution study of (NBS) HAP (sample B) crystals in partially saturated acidic media. Bulk solution conditions $0.1 M$ acetate buffer at $\mathrm{pH}$ of 4.5 and $0.5 \mathrm{M}$

identical, showing no unusual transport behavior under conditions that are similar to those with the HAP slurries.

The bag constant, $\mathrm{K}$, was found to be 1.56 $\times 10^{-2} \mathrm{~min}^{-1}$ from these experiments corresponding to a half-life of about 44 minutes.

DiAlysis bag RESUltS FOR HAP DISSOLUTION.-Figure 4 shows the results of a typical experiment with HAP crystals in the dialysis bag when calcium ion concentration was determined by atomic absorption spectrophotometry. The upper points are the ambient solution concentrations inside the bag and the lower points ionic strength $\mathrm{TCa}=\mathrm{TP}=8.536 \times 10^{-3} M$. Solid circle in square, inside bag (7.8 mg HAP in $20 \mathrm{ml}$ bulk solution).

are the solution concentrations outside the bag. Note the unusual oscillatory pattern of the ion concentration variation inside the bag. Sample A (authentic [NBS] HAP) was used in this experiment and, except for a few runs involving the other two samples, in all of the other experiments conducted in this study. The pattern shown in Figure 4 represents a determination from a relatively large background. However, these results have been highly reproducible both with regard to the peak-to-valley concentration differences and the times at which the peaks and valleys occur. Although under usual favor- 
able conditions the atomic absorption analysis for calcium is accurate to only around $\pm 1 \%$, the repeatability of consecutive readings for the results seen in Figure 4 was around $0.3 \%$. Therefore, the "signal-to-noise" ratio for these results should be around 10 to 20 .

Figure 5 is a typical set of the results obtained using sample $\mathrm{C}$ ( ${ }^{45} \mathrm{Ca}$-labeled HAP). In these experiments, the partially saturated bulk solution used was of the same specific activity as that of sample C. Note the good agreement between the concentration based on the phosphate analysis (colorimetric) ${ }^{10}$ and the ${ }^{45} \mathrm{Ca}$ analysis (scintillation counter).* This shows that good congruency was maintained throughout the experiment. Congruency was always observed in all the other experiments conducted in this study.

* Beckman Liquid Scintillation System, Beckman Instruments, Inc., Fullerton, $\mathrm{Ca}$.

f Activity products were calculated using the computer program developed by Fox and Higuchi..11
Figure 6 shows a typical set of data obtained using sample B (cold [NSB] HAP). These results are in good agreement with the runs obtained with the other two samples. The oscillations of ion concentrations in the bag strongly support the synchronized dissolution hypothesis that the HAP crystals are dissolving in phase, so to speak, bursting ions periodically with a diffusional relaxation period in between (Fig 1).

The data in Figure 4, for example, may be interpreted in the following way. Initially $10<$ $t<40$ ), there is only diffusion of the ions from inside the bag to the outside solution with no significant crystal dissolution taking place. The decay rate during this period is quantitatively consistent with this interpletation, that is, since the bag time constant is the order of 44 minutes (Fig 3). Around $\mathfrak{t} \simeq 40$ minutes, the activity product $f$ of the inside solution drops to around $1 \times 10^{-122}$ and this "triggers" a brief dissolution

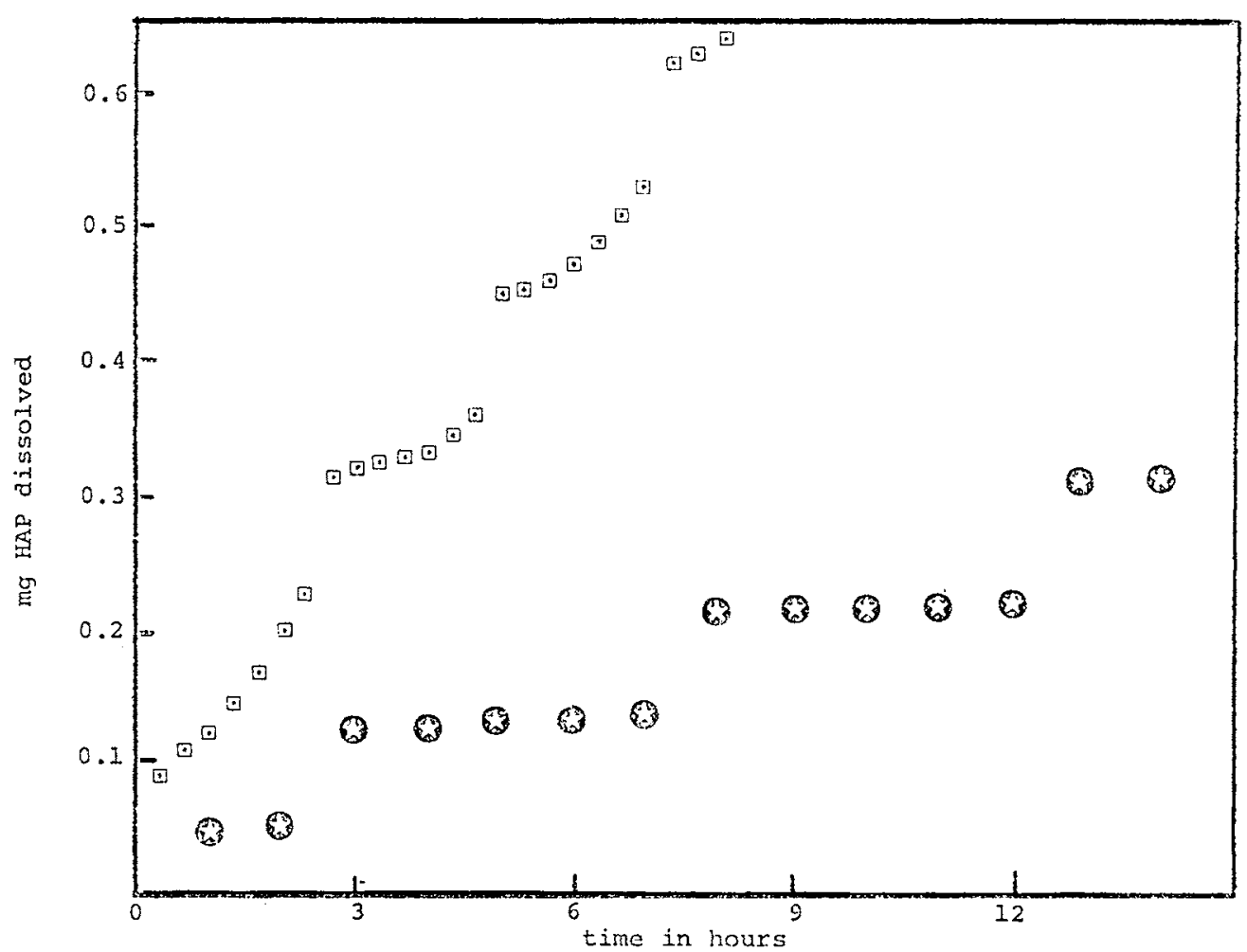

FIg 7.-Dissolution rate behavior of human dental enamel block $\left(A=0.125 \mathrm{~cm}^{2}\right)$ in partially saturated acetate buffer at $500 \mathrm{rpm}$ (rotating disk). Bulk solution conditions: 0.1 $M$ acetate buffer at $\mathrm{pH}$ of 4.5 and $0.5 \mathrm{M}$ ionic strength. Open star in solid circle: $\mathrm{TCa}=4.69$ $\times 10^{-2} M ; \mathrm{TP}=4.69 \times 10^{-4} M$. Solid circle in open square: $\mathrm{TCa}=1.98 \times 10^{-2} \mathrm{M} ; \mathrm{TP}=$ $1.98 \times 10^{-4} \mathrm{M}$. 


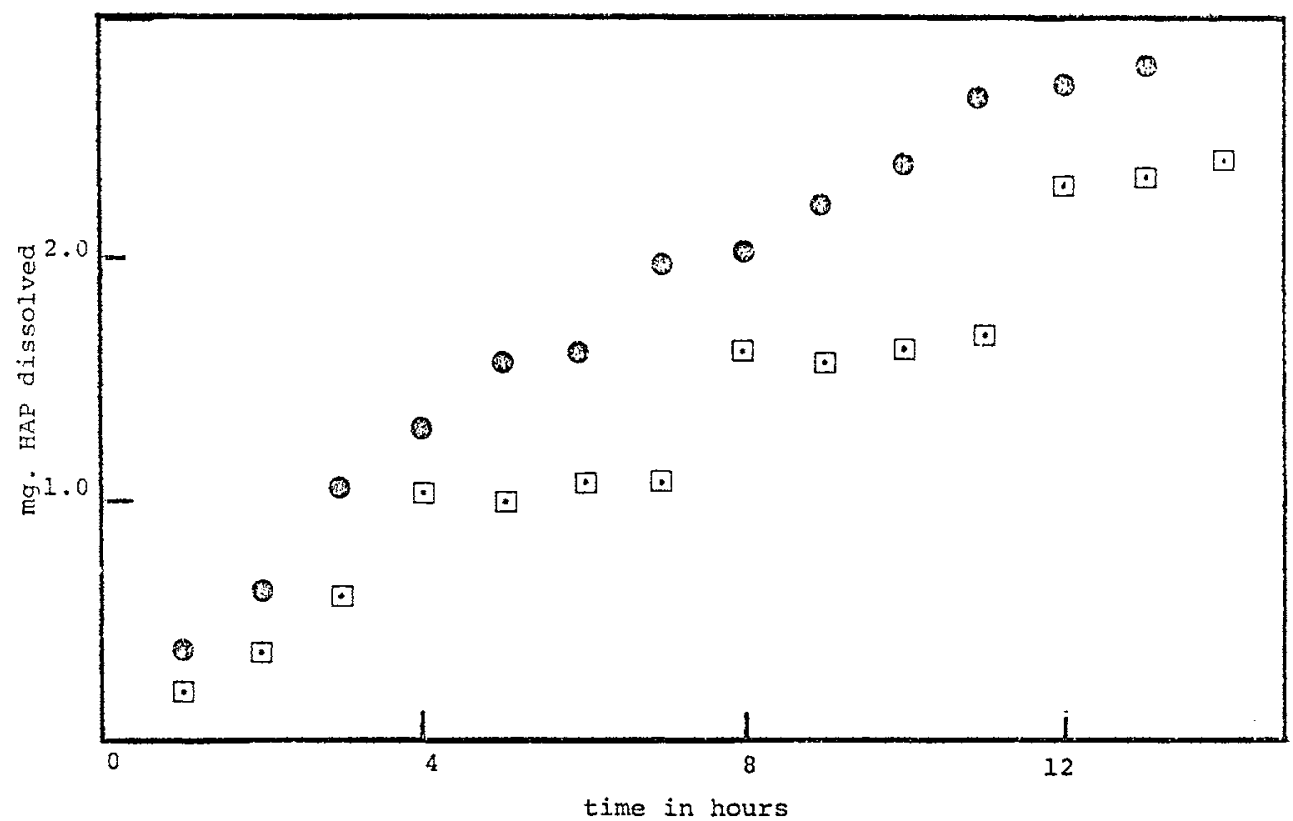

FIG 8.-Dissolution rate behavior of (NBS) HAP pellets $\left(A=0.317 \mathrm{~cm}^{2}\right)$ in partially saturated acetate buffer solution at $150 \mathrm{rpm}$ (static disk). Bulk solution conditions: $0.1 M$ acetate buffer at $\mathrm{pH}$ of 4.5 and $0.5 \mathrm{M}$ ionic strength. Solid circle, TCa $=1.98 \times 10^{-2} M$; TN $=1.98$ $\times 10^{-3} \mathrm{M}$. Solid circle in open square, $\mathrm{TCa}=$ $8.356 \times 10^{-3} M ; \mathrm{TP}=8.356 \times 10^{-4} \mathrm{M}$. period, eliciting a peaking of the ion concentrations inside the bag. During this short period, it is believed that essentially all crystals dissolve a domainal increment, further dissolution being inhibited by the rise in the ambient ion concentration as described in the theoretical section. Calculations show that the increment represents about $5 \%$ of the crystal. This amount could be associated with an increment of the order of dimensions of a unit cell if the dissolution takes place in directions perpendicular to the dominant planes of the crystals or it may be associated with dissolution of several unit cells if the dissolution is occurring, for example, in directions perpendicular to the C-plane of the crystal. That the dissolution takes place as synchrcnized bursts from all crystals during a very brief period is consistent with the second, third, fourth, etc. decay portions of the pattern, being essentially the same and consistent, with only diffusional relaxation taking place. If significant crystal dissolution werc occurring during the decay period, the frequency of the oscillation would have been significantly less than that observed. Each time when the inside solution concentration drops to a critical point (that is, $\mathrm{K}_{\mathrm{HAP}}=\left(\mathrm{a}_{\mathrm{Ca}^{2}}{ }^{2+}\right)^{10} \quad\left(\mathrm{a}_{\mathrm{PO}}^{3-}\right)^{6} \quad\left(\mathrm{a}_{\mathrm{OH}^{-}}\right)^{2}=$ $\left.10^{-122}\right)$, a synchronized burst takes place and the process is repeated.

To demonstrate that the involvement of other calcium phosphate phases (such as dicalcium phosphate dihydrate) was not possible in these experiments, $\mathbf{K}_{\mathrm{HAP}}$ calculations were done $\mathrm{e}^{11}$ for solution compositions corresponding to the peaks. The calculations showed that even at the peaks the systems were substantially undersaturated with respect to all phases (for example, about one-third to one-half saturation with respect to dicalcium phosphate dihydrate). These theoretical results were also verified experimentally by demonstrating that the supernatant solution at the peaks readily dissolved added dicalcium phosphate dihydrate crystals.

In addition to supporting the proposed mechanism, the experiments are also consistent with the acid buffer dissolution data obtained ${ }^{1}$ with a block section of human dental enamel and compressed pellets of synthetic HAP under similar conditions, that is, under substantial partial saturation conditions. Figures 7 and 8 show data taken from a recent study. ${ }^{1}$ The 
unusual stepwise dissolution patterns are consistent with the present results using the dialysis bag system. The oscillatory patterns obtained in the dialysis bag experiments may be considered to be the mathematical derivative of the stepwise pattern shown in Figures 7 and 8. The

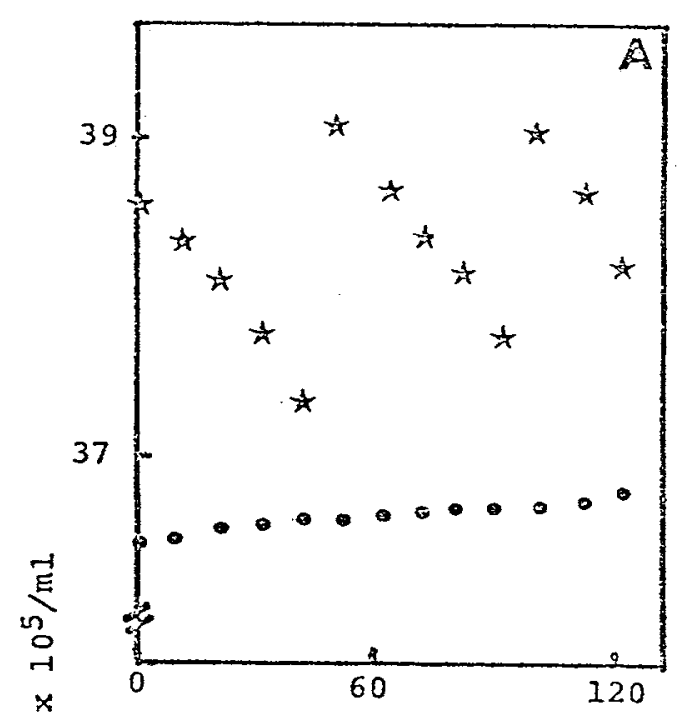$$
\text { 琣 }
$$

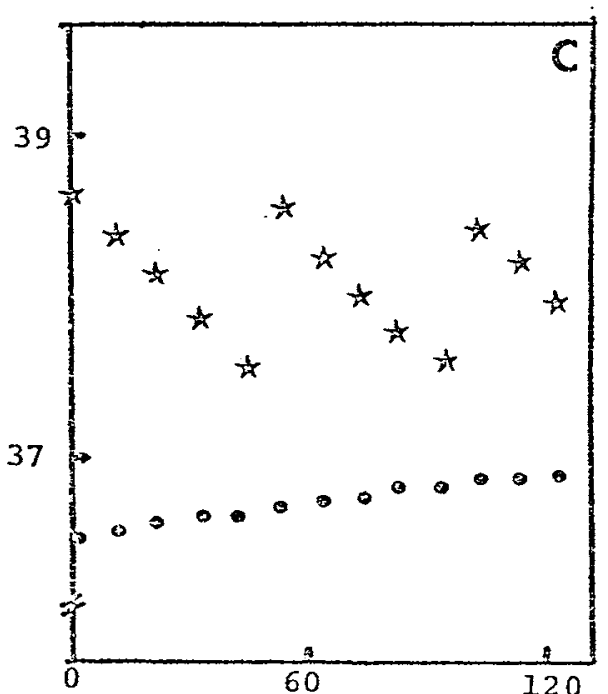

plateau regions in Figures 7 and 8 would correspond to the diffusional relaxation (that is, the decay) regions of Figure 4, for example, and the step "jumps" in the former would correspond to the peaks in Figure 4.

Additional studies to EXamine the
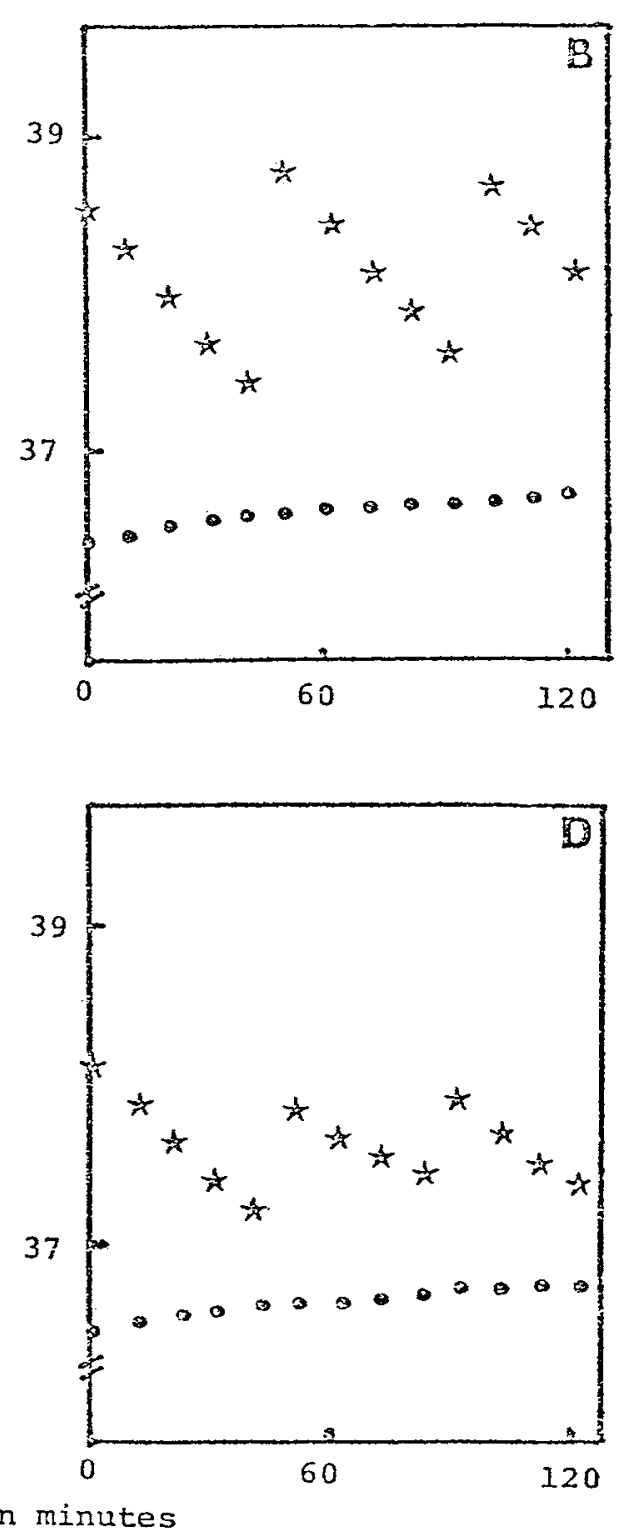

FIG 9.-Influence of slurry density on dialysis transport-dissolution study of (NBS) HAP (sample A) crystals in partially saturated acidic media. Bulk solution conditions: $0.1 \mathrm{M}$ acetate buffer at $\mathrm{pH}$ of 4.5 and $0.5 \mathrm{M}$ ionic strength,

$\mathrm{TCa}=\mathrm{TP}$. Solid star, inside bag $(A, 9 \mathrm{mg} \mathrm{HAP}$ in $20 \mathrm{ml}$ bulk solution; $B, 7 \mathrm{mg} \mathrm{HAP}$ in $20 \mathrm{ml}$; $C, 6 \mathrm{mg}$ HAP in $20 \mathrm{ml}$; and $D, 3 \mathrm{mg}$ HAP in $20 \mathrm{ml}$ bulk solution). Solid circle, outside bag. 
PROPOSED MEGHANISM.-Influence of changing the slurry density.--According to the proposed mechanism, a constant increment of dissolution from each crystal should be involved during the burst period. Therefore, the amount of crystals present in the dialysis bag would be expected to determine the oscillatory peak heights if the model is correct. In view of this, experiments were conducted in which different slurry densities of HAP were used under otherwise iden-

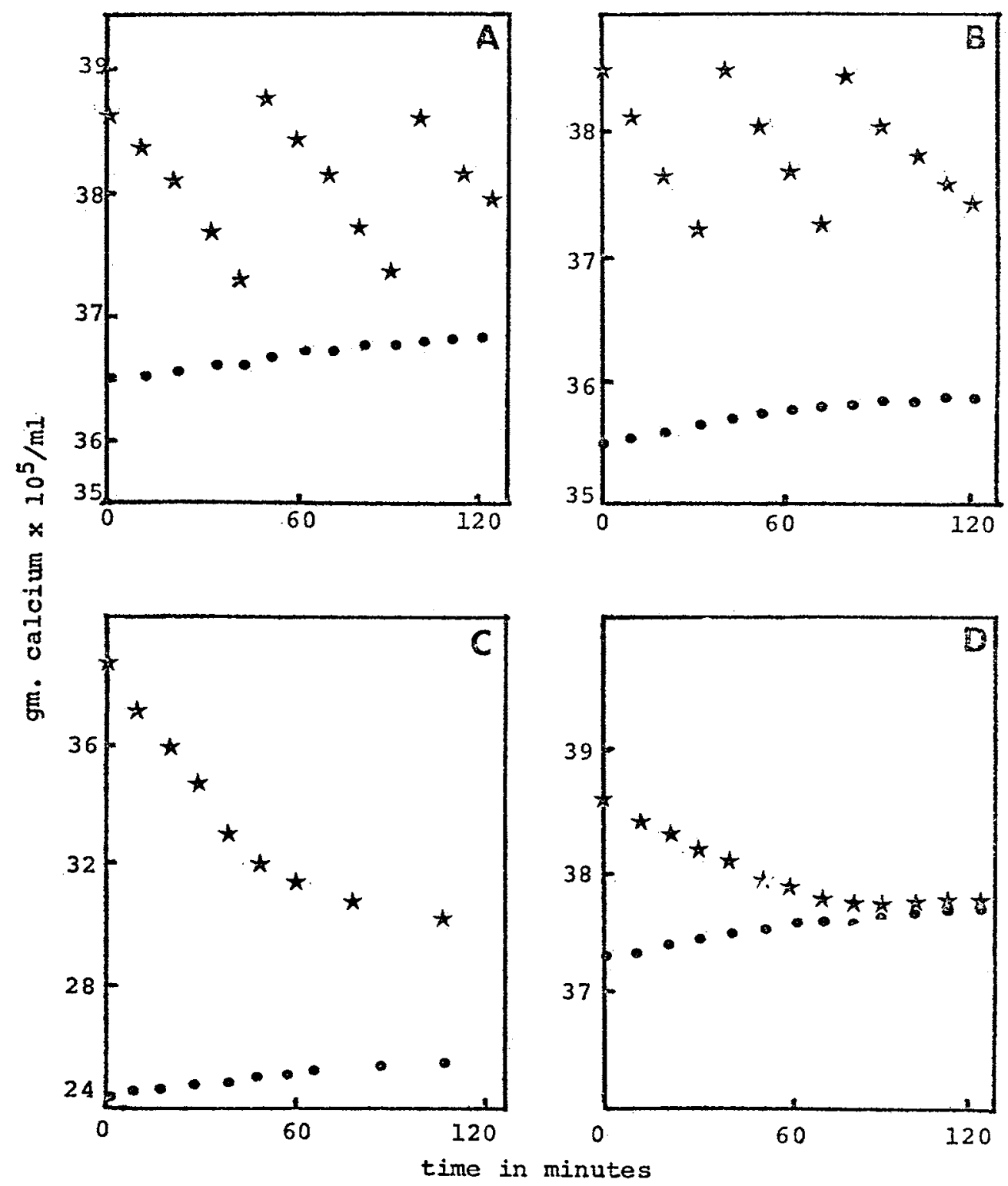

FIG 10.--Influence of $\triangle \mathrm{C}$ (concentration differential) on dialysis transport-dissolution of (NBS) HAP (sample A) crystals in partially saturated acidic media. Bulk solution conditions: all slurries in bag prepared by suspending $8 \mathrm{mg}$ of HAP in $20 \mathrm{ml}$ of solution $0.1 \mathrm{M}$ acetate buf-

fer at $\mathrm{pH} 4.5$ and ionic strength of $0.5 \mathrm{M}$, and $\mathrm{TCa}=\mathrm{TP}=8.54 \times 10^{-3} M$. For outside partially saturated solutions: $0.1 M$ acetate buffer at $\mathrm{pH}$ of 4.5 and $0.5 \mathrm{M}$ ionic strength and $\mathrm{TCa}=\mathrm{TP}$ for all solutions. Solid star, inside bag; solid circle, outside bag. 
tical conditions. Figure 9 shows that changing the slurry density inside the bag from 3 to 9 $\mathrm{mg} / 20 \mathrm{ml}$ proportionately increased the ion concentration peak heights as predicted by the model.

Influence of changing the concentration differential between inside and outside the dialysis bag.--Figure 10 shows the results of experiments in which the concentration differential, $\Delta \mathrm{C}$, between inside and outside the bay was changed. When $\Delta \mathrm{C}$ was increased by 30 to $40 \%$, the experiment showed an approximate proportional increase in the frequency of the oscillations following in good agreement with the model predictions as shown in Figure 10,B. Setting $\Delta \mathrm{C}$ to a relatively large value resulted in a very rapid decay in the ion concentration inside the bag and the oscillatory pattern was not observed (Fig 10,G). This could be the result of the drop in the ambient solution activity product being too rapid and not allowing the crystals to synchronize. Thus, desynchronized or steady dissolution of the crystals takes place under these conditions. Finally, Figure 10,D, shows the results of an experiment when the $\Delta \mathrm{C}$ is made too small for the ambient solution concentration to drop below the critical activity product during the diffusional relaxation period. Thus, only the initial transient decay is observed in this instance.

Influence of changing the stirring rate inside and outside the dialysis bag.-The possibility of any influence of the stirring speed on the oscillation phenomenon was examined by running the same experiments, except for the stirring speed ( 600 to $150 \mathrm{rpm}$.). There was no noticeable difference between the results of the two experiments. Thus, this experiment showed that there was no significant buildup of boundary layers around the crystals or the crystal aggregates in the dialysis bag.

Influence of temperature.- The results obtained when the experiment was conducted at $37 \mathrm{C}$ showed no apparent difference from those obtained at $30 \mathrm{C}$. The implications of this from the in vivo standpoint are apparent.

\section{Conclusions}

The synchronous dissolution hypothesis for HAP crystal dissolution under high partial saturation conditions has been experimentally tested by following the solution ion concentration of HAP suspension in dialysis bags immersed in calcium phosphate solutions. In ac- cordance with the hypothesis, oscillations of the ion concentrations in the dialysis bags were observed for a wide range of conditions. The data obtained from these studies are consistent with the repetitive stepwise dissolution behavior of both human dental enamel blocks and compressed HAP pellets in solutions of high partial saturation.

A crystal model has been proposed based on an effective surface solubility variation as dissolution proceeds through domains having dimensions of the order of the unit cell. It is believed that a $\mathrm{K}_{\mathrm{HAP}}$ of about $1 \times 10^{-122}$ corresponds to the ion activity product for the lowest "solubility" plane in the dissolving domain and it is associated with the "firing" point for the oscillation behavior. This ion activity product is essentially the same as that for site No. 1 of the recently developed ${ }^{12}$ two-site model for HAP dissolution under low-tomoderate partial saturation conditions. The present study together with other recent investigations ${ }^{8}$ provide, for the first time, a rather complete picture of tooth enamel dissolution behavior under a very wide range of conditions. These findings are also consistent with the many interesting and heretofore puzzling features of enamel and HAP dissolution.

It is also believed that the result of the present study should be of significant importance in an eventual understanding of the anomalous solubility behavior of HAP, ${ }^{4-6}$ for example, the slurry density effect on the solubility and the very long times required before reaching solubility equilibrium. According to the proposed model, the usually slow rate of reaching equilibrium in powdered apatite solubility experiments may be explained by the crystal population being out-of-phase (Fig 1) at high slurry densities where there may be some crystals with unit cells dissolving and some crystals with their unit cells "growing" to reach the same chemical potential. As this process is analogous to the simultaneous dissolution of one phase and the formation of another, the driving forces involved could be relatively small and therefore, the rates for reaching equilibrium would be much slower than in simple onephase dissolution.

\section{References}

1. Fawzi, M.B.; Young, F.; Higuchi, W.I.; and Hefrerren, J.J.: Unusual Dissolution Behavior of Enamel in Partially Saturated Acidic Media, J Dent Res 53(Sperial Issue): Abstract No. 868, 1974. 
2. Wu, M.S.; Higuchi, W.I.; Dedhiya, M.G.; Shimabayas hi, S.; and Hefferren, J.J.; Rotating Disk Method Studies of Dental Enamel and Synthetic Hydroxyapatite Dissolution, J Dent Res 54(Special Issue A): Abstract No. L538, 1975.

3. Higuchi, W.I.; Gray, J.A.; HefFerren, J.J.; and PATEL, P.R.: Mechanisms of Enamel Dissolution in Acid Buffers, $]$ Dent Res 44:330-340, 1965.

4. Levinskas, G.J., and Neuman, W.F.: The Solubility of Bone Mineral: I. Solubility Studies of Synthetic Hydroxyapatite, $J$ Phys Chem 59:164-168, 1966.

5. Rootare, H.M.; Dietz, V.R.; and CarPENTER, F.G.: Solubility Product Phenomena in Hydroxypatite-Water System, J Colloid Sci 17:170-206, 1962.

6. Clark, J.S.: Solubility Criteria for the Existence of Hydroxyapatite, Can J Chem 33: 1696-1700, 1955.

7. Darling, A.I.: Mechanisms of Hard Tissue Destruction, Sognnaes, R.F. (ed), Wash- ington, DC: Am Assoc Advan Sci, pp 171$186,1963$.

8. FawzI, M.B.; Sonobe, T.; Higuchi, W.I.; Hefferren, J.J.: Proposed Mechanism for Hydroxyapatite Dissolution Under Partial Saturation: Synchronized Crystal Dissolution, $J$ Dent Res 54(Special Issue A):Abstract No. 449, 1975.

9. Moreno, E.C.; Gregory, T.M.; and Brown, W.E.: Preparation and Solubility of Hydroxyapatite, J Res NBS, 72A: 773, 1968.

10. Amin, K.N.: Solubility of Hydroxyapatite and Its Inhibition by Phosphate, PhD thesis, University of Michigan, 1971.

11. Fox, J.S., and Higuchi, W.I.: Methodologies for Transport Calculations Involving Diffusion, Complex Chemical Equilibria and Crystal Kinetics, $J$ Dent Res, in press.

12. Fox, J.L.; FawzI, M.B.; Wu, M.S.; and Higuchi, W.I.: A New Two-Site Model for Hydroxyapatite Dissolution in Acid Buffers, Arch Oral Biol, in press. 